Research Article

\title{
Numerical Modelling of Two-Phase Flow in a Gas Separator Using the Eulerian-Lagrangian Flow Model
}

\author{
S. Amzin $(\mathbb{D}$, S. Norheim, B. Haugen, B. Rødland, and H. Momeni \\ Western Norway University of Applied Sciences, Department of Mechanical and Marine Engineering, Bergen N5020, Norway \\ Correspondence should be addressed to S. Amzin; swed526@gmail.com
}

Received 4 December 2019; Revised 3 December 2020; Accepted 3 May 2021; Published 20 May 2021

Academic Editor: Oronzio Manca

Copyright ( $\odot 2021$ S. Amzin et al. This is an open access article distributed under the Creative Commons Attribution License, which permits unrestricted use, distribution, and reproduction in any medium, provided the original work is properly cited.

Gravity-driven separators are broadly used in various engineering applications to remove particulate matters from gaseous fluids to meet legislation demands. This study represents a detailed numerical investigation of a two-phase cyclone separator using the Eulerian-Lagrangian gas flow method. The turbulence is modelled using the Reynolds stress model (RSM). The technique has successfully predicted the typical trends and variations seen in such gas separators with an average error of approximately $5.5 \%$. Also, the computed results show a realistic agreement with the experimental measurements.

\section{Introduction}

Air pollution, equally natural and artificial pollution, is one of the conventional environmental dilemmas known to humankind. However, with the emergence of the Industrial Revolution in the 18th and 19th centuries, the phenomenon continues to deteriorate, and air pollution becomes one of the most complex environmental challenges. The health effects of air contamination are severe; for instance, air pollution due to particulate matter (PM), nitrogen dioxide, and ozone is a significant cause of severe health problems $[1,2]$. Thus, environmental legislation has become more stringent to improve air quality and is forcing engineers to develop an efficient industrial system.

Cyclones are one of the widely used devices in many industries for separating substances with different densities. The selection of separators is driven by their simple construction and low operating and maintenance costs. The separation is achieved by employing the centrifugal and the gravitational forces, which generates a cyclonic vortex $[3,4]$. The cyclone is often part of a complete air purification unit. The large-sized particulates are removed from the gas stream in the cyclone before being directed through other filtration devices to remove the fine particulates. Traditionally, separators are classified based on their geometry as vertical, horizontal, and spherical, where each has its advantages and disadvantages [4]. However, several factors must be considered when selecting an industrial separator for a given application, for instance, among those, the traits of the fluid to be processed, size, transportation, and the cost.

The untreated gas enters tangentially from the inlet at high velocity in vertical separators due to the centripetal forces. It continues to flow spirally to the lower part of the cyclone. To be noted is that the higher the tangential velocity, the higher the centrifugal forces. Due to the gradual reduction in the separator's cone, the gas velocity increases, creating an additional inner central vortex at the separator's centre-the inner vortex flows upward, carrying the clean gas [5]. Eventually, the separated particles exit at the separator's bottom because of their high density [3].

The cyclone geometry and operational parameters have a vital influence on separation performance and collection efficiency [6-8]. Operating parameters such as particle diameter and density, pressure drop, and the inlet velocities are of interest. For example, a lower tangential velocity is regularly linked to an increase in the cyclone inlet dimensions. Ultimately, the reduction in the tangential velocity leads to the degradation of collection efficiency. Experimental studies have shown that increasing the inlet velocity will affect the flow pattern and results in a higher pressure drop [9]. 
Among the geometric parameters that affect the separation performance are the dimensions of the separator cylinder, cone, inlet and outlet, and vortex finder [5, 8]. Experimental studies have shown that the vortex finder's dimensions significantly influence the pressure drop, e.g., increasing the vortex finder length will increase the pressure drop [10]. The impact of the vortex finder diameter on the cyclone performance is also studied in [6-8]. The separation efficiency was higher for a smaller diameter for the vortex finder because of higher tangential velocity and higher centrifugal forces impinging the particles towards the cyclone's wall.

Furthermore, the cone dimensions impact the cyclone performance, as addressed in [11]. It is achievable to enhance the efficiency of the separation and the gas flow's symmetry by adding a second spiral inlet to the cyclone [12]. The separation efficiency also decreases with the reduction of the outlet diameter because particles are easily caught in the vortex instead of escaping through the outlet [5]. Additionally, the separator's conical part greatly influences the residence time of the particles because it increases the centripetal force acting on the particles. Hence, a smaller conical section leads to a lower collection efficiency because it will be more difficult for the vortex to capture the particles and carry them up towards the top outlet [5].

The effect of the added components inside the cyclone has also been studied experimentally and numerically $[7,13,14]$. The separator has an outer cylinder, a metal rod, and a vortex limiter-the metal rod increases the tangential velocity, centrifugal forces, and efficiency [7]. The study indicates that the vortex limiter promotes higher collection efficiency and heat rate for a given particle size [8]. An insignificant pressure drop has also been noticed.

Advanced modelling techniques are widely used in many engineering applications to design or/and optimise practical systems [15]. They are efficient, cost effective, and can produce detailed information that is sometimes challenging and costly to produce by experiments. Computational fluid dynamics (CFD) is vital in optimising separation equipment. It is capable of predicting separation efficiency and overall performance. However, the accuracy of CFD analysis depends on the accuracy of its built-in physical models. Therefore, validation of existing numerical models is significant to support their evaluation and improvement.

The prime objective of this study is to carry out a detailed numerical analysis using the Eulerian-Lagrangian [16] gas flow model and compare the results with experimental measurements [17].

\section{The Eulerian-Lagrangian Gas Flow Model}

In this section, the Eulerian-Lagrangian method, which is used to observe and analyse the separator's fluid flow, is discussed briefly.
Fluid flows can be analysed mathematically, either using the Lagrangian description where the trajectories of the individual fluid particles are tracked in time [16] or/and using the Eulerian representation where the evolution of the fluid properties is observed at every point in space as time varies [18]. It is noted that it can be computationally expensive to track all the fluid particles in a flow; therefore, only selected particle trajectories are tracked.

In multiphase fluids, both descriptions are combined where the gas phase is solved in conjunction with tracking individual particles. The particles are tracked by indirectly solving transport equations using the Lagrangian particle method. The conservation of mass and momentum is represented by Eulerian conservation equations [19].

2.1. Gas-Phase Flow. The equations of mass and momentum conservation are solved for the continuous phase, which is the gas phase.

$$
\frac{\partial \bar{\rho}}{\partial t}+\frac{\partial \bar{\rho} \tilde{u}_{i}}{\partial x_{i}}=0
$$

The first term indicates the time variation, and the second term shows the changes due to fluid transport. $\rho$ is the density of the gas, and $u$ is the average velocity of the gas.

$$
\frac{\partial\left(\bar{\rho} \tilde{u}_{i}\right)}{\partial t}+\frac{\partial\left(\bar{\rho} \tilde{u}_{i} \tilde{u}_{j}\right)}{\partial x_{i}}=\frac{\partial \bar{P}}{\partial x_{j}}+\frac{\partial}{\partial x_{i}}\left(\tau_{i j}-\rho \overline{u_{i}^{\prime} u_{j}^{\prime}}\right)+\overline{F_{i}} \text {. }
$$

The first term on the LHS indicates the unsteady term, while the second term indicates the rate of change. On the RHS, the first term indicates the pressure gradient, where $P$ is the pressure. The second term means the momentum due to viscous forces, where $\tau_{i j}$ is the viscous stress tensor and $\rho \overline{u_{i}^{\prime} u_{j}^{\prime}}$ is the Reynolds stress term. The third term is the coupling term between the phases, and it approximates the sum of the drag on each particle occurring inside a fluid control volume.

The dominating swirl inside the separator creates an anisotropic turbulence field. When modelling the turbulence field, the Reynolds stress model (RSM) is adopted [20]. The RSM is second-moment closures where the eddy-viscosity hypothesis is avoided. It solves the individual components of the Reynolds stress tensor (six equations) instead of two similar to $k$-epsilon or $k$-omega. This model is more computationally expensive but is more accurate because it captures the complex interactions in turbulent flows.

The RSM provides differential transport equations for each of the Reynolds stress components.

$$
\frac{\partial\left(\rho \overline{u_{i}^{\prime} u_{j}^{\prime}}\right)}{\partial t}+\frac{\partial\left(\rho \tilde{u} \overline{u_{i}^{\prime} u_{j}^{\prime}}\right)}{\partial x_{k}}=D_{T, i j}+D_{L, i j}+P_{i j}+G_{i j}+\phi_{i j}+\varepsilon_{i j} \text {. }
$$


$D_{T, i j}$ represents the turbulent diffusion, $D_{L, i j}$ is the molecular diffusion, $P_{i j}$ is the stress production, $G_{i j}$ is the buoyancy production, $\varphi_{i j}$ is the pressure strain, and $\varepsilon_{i j}$ is the dissipation. These terms are a function of the mean gasphase velocity gradients.

$$
\begin{aligned}
D_{T, i j}= & -\frac{\partial}{\partial x_{k}}\left[\rho \overline{u_{i}^{\prime} u_{j}^{\prime} u_{k}^{\prime}}+\overline{\rho^{\prime}\left(\delta_{k j} u_{i}^{\prime}+\delta_{i k} u_{j}^{\prime}\right)}\right], \\
D_{L, i j}= & \frac{\partial}{\partial x_{k}}\left[\mu \frac{\partial}{\partial x_{k}} \overline{\left(u_{i}^{\prime} u_{j}^{\prime}\right)}\right] \\
P_{i j}= & -\rho\left(\overline{u_{i}^{\prime} u_{k}^{\prime}} \frac{\partial u_{j}}{\partial x_{k}}+\overline{u_{j}^{\prime} u_{k}^{\prime}} \frac{\partial u_{i}}{\partial x_{k}}\right), \\
G_{i j}= & -\rho \beta\left(g_{i} \overline{u_{j}^{\prime} \theta}+g_{j} \overline{u_{i}^{\prime} \theta}\right), \\
\phi_{i j}= & p^{\prime}\left(\frac{u \partial_{i}^{\prime}}{\partial x_{j}}+\frac{\partial u_{j}^{\prime}}{\partial x_{i}}\right) \\
\varepsilon_{i j}= & -2 \mu \frac{\partial \frac{u_{i}^{\prime}}{\partial x_{k}} \overline{\partial u_{j}^{\prime}} .}{\partial x_{k}}
\end{aligned}
$$

2.2. Lagrangian Particles' Tracking. The Lagrangian method is based on a local force balance on each particle. The force balance considers the particle inertia with the forces acting on it and can be expressed as

$$
\frac{d u_{p}}{d t}=\frac{u_{g}-u_{p}}{\tau_{r}}+\frac{g\left(\rho_{p}-\rho_{g}\right)}{\rho_{p}}+F .
$$

The LHS represents the inertial force per unit mass, where $u_{\mathrm{p}}$ is the particle velocity. On the RHS, the first term expresses the drag between the phases, where $u_{\mathrm{g}}$ is the gas phase velocity. The second term represents gravity and buoyancy, respectively. $g$ is the gravity constant, and $\rho_{p}$ and $\rho g$ are the density of the particle and air, respectively. The last term represents any additional forces that may act upon the particles. $\tau_{r}$ represents the particle relaxation time and is expressed as

$$
\begin{aligned}
\tau_{r} & =\frac{\rho_{p} d_{p}^{2}}{18 \mu} * F_{d}, \\
F_{d} & =\frac{24}{C_{d} * \operatorname{Re}},
\end{aligned}
$$

where $d_{p}$ is the particle diameter, $\mu$ is the molecular viscosity of the fluid, $F_{d}$ is the drag force, $C_{d}$ is the drag coefficient, and $\mathrm{Re}$ is the relative Reynolds number, which is defined as

$$
\operatorname{Re} \equiv \frac{\rho_{g} d_{p}\left|u_{p}-u_{g}\right|}{\mu} .
$$

One-way coupling is used in the simulations, which means that the fluid phase influences the particles via aerodynamic drag. For the drag coefficient, $C_{d}$, the Schiller-Naumann model is used [21].

$$
C_{d}= \begin{cases}\frac{24\left(1+0,15 \mathrm{Re}^{0,687}\right)}{\operatorname{Re}}, & \mathrm{Re} \leq 1000, \\ 0,44, & \mathrm{Re}>1000 .\end{cases}
$$

\section{Experimental and Numerical Setup}

The computational results obtained by the CFD analysis are compared to the experimental results of Wang et al. [17]. The experiment is conducted using air contaminated with cement particles. The contaminated air is blown into a vertical separator at a velocity of $20 \mathrm{~m} / \mathrm{s}$. A flow meter is used to measure the flow rate. The outlets are open to the air at a pressure of $1 \mathrm{~atm}$. The particle phase volume fraction is less than $10 \%$. The density of the particles is $3320 \mathrm{~kg} / \mathrm{m}^{3}$. A probe is used to measure the velocity and pressure of the gas field. It is placed in the flow field with five pressure transducers to obtain voltage signals. The particle distribution of the cement material can be expressed by the Rosin-Rammler equation [22].

$$
R(d)=e^{-\left(d^{n} / \bar{d}\right)}
$$

where $R(d)$ is the mass fraction of particles with a diameter greater than $d . \bar{d}$ equals to the characteristic diameter; in this case, it is equal to $29.90 \mu \mathrm{m}$. $n$ is the distribution parameter and is specified to be 0.806 .

A schematic of the cyclone, along with the selected axial locations, is shown in Figure 1. Each axial location spans $175 \mathrm{~mm}$ in length. The computational parameters used in this study are presented in Table 1 . The boundary conditions for the inlet are set to be as the velocity inlet, while both outlets are set to be as the pressure outlet.

The computational tool used in this study is commercial software STAR-CCM+. The tool solves the steady Favreaveraged transport equations (1)-(3) and (10) along with their selected closures on the physical grid. The nonlinear differential steady governing equations for the complex flow fields are discretised using a mixed finite element method, which employs stabilisation techniques to address issues with the pressure-velocity coupling and the nonlinear convection terms.

The Reynolds stress turbulence model (RSM) is used because of the intense swirl inside the separator. Since the volume fraction of the particles is low, a point-particle injector is used to add particles. To be noted is that the computational model selected for the two-phase simulation captures physics and the typical behaviour of such separators reasonably. Hence, the chosen computational model is independent of the chosen boundary conditions shown in [17].

The physical grid is refined near the centre line near the outlet and inlet streams to capture the details with a reasonable computational cost. The sensitivity of the computed solution to the cell size and type is examined by using two different types of cells (hexahedrons and polyhedrons). It 

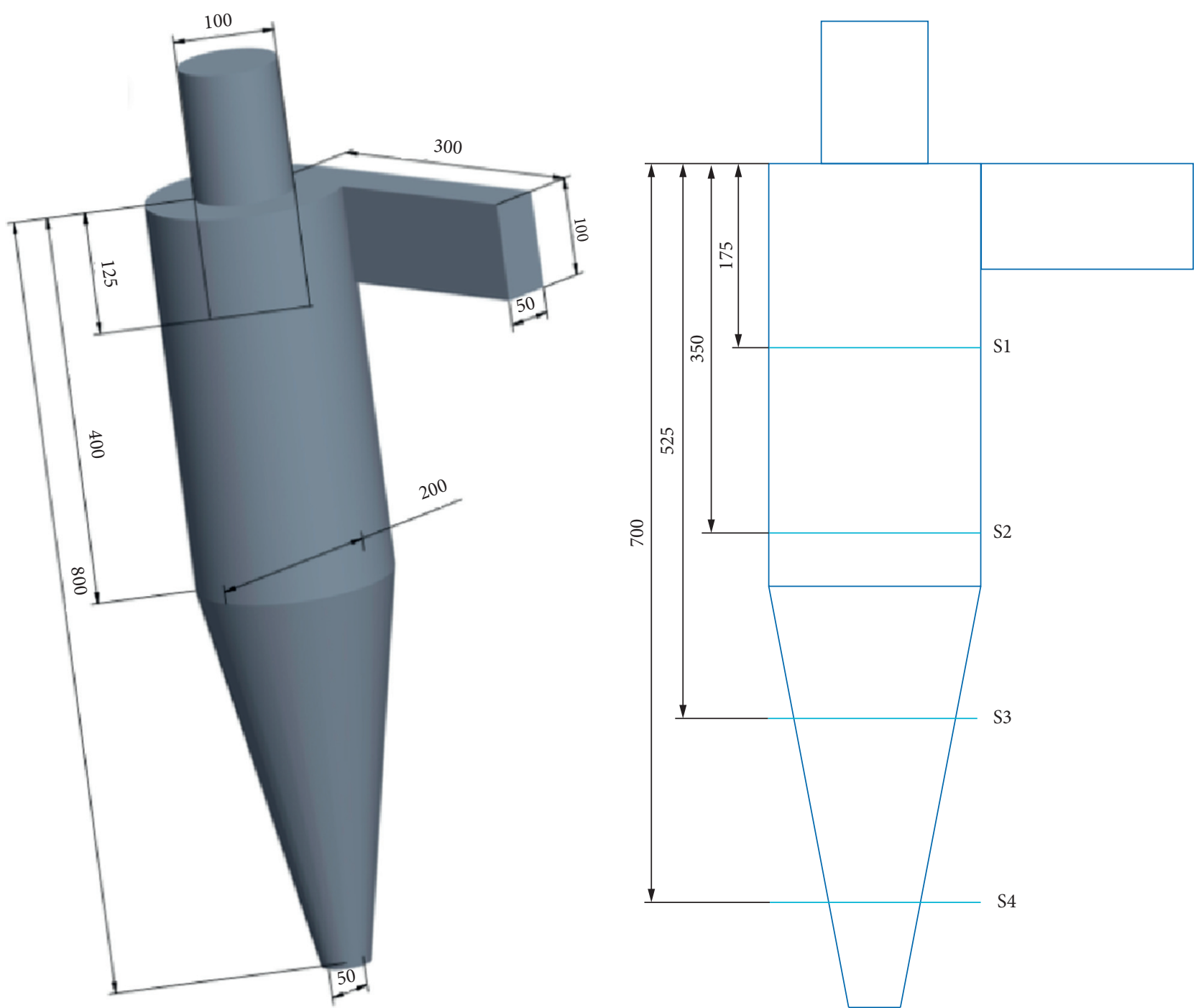

Figure 1: Schematic and the axial sections of the test cyclone [16].

TABLE 1: Boundary conditions.

\begin{tabular}{lc}
\hline Density of air & $1.205 \mathrm{~kg} / \mathrm{m}^{3}$ \\
Density of particles & $3320 \mathrm{~kg} / \mathrm{m}^{3}$ \\
The volume fraction of the discrete phase & $3 \%$ \\
Pressure & $1 \mathrm{bar}$ (atmospheric) \\
Temperature & $300 \mathrm{~K}$ \\
Inlet velocity & $5-35 \mathrm{~m} / \mathrm{s}$ \\
Particle diameter & $1-5 \mu \mathrm{m}$
\end{tabular}

has been observed that the polyhedron cells produce a more realistic solution. The polyhedral mesh is less sensitive to stretching since the gradients can be accurately approximated as each of the individual cells has sufficient neighbouring cells.

The flow is set to be implicit unsteady, with a time step of 0.01 seconds. The turbulence intensity is set to 0.01 . The solution has converged in approximately 6000 iterations. Hence, the solution presented in Section 4 shows a negligible grid sensitivity and thus is fully converged. The sensitivity test is summarised in Table 2. The conversion residual is shown in Figure 2.
TABle 2: Grid sensitivity test.

\begin{tabular}{lcc}
\hline Cell type & Cells & Pressure \\
\hline Poly & 39266 & 1417 \\
Poly & 99436 & 1433,1 \\
Poly & 19887 & 1538,7 \\
Hexa & 187558 & 1005,9 \\
\hline
\end{tabular}

\section{Results and Discussion}

The pressure drop is a vital parameter in industrial separators. For a higher pressure drop, greater power is required to move the fluid across the separator. Figure 3 shows the computed correlation between velocity and pressure drop inside the separator. Typically, the pressure drop increases with increasing inlet velocity. Although it is observed that the computed results are slightly lower than the measured results for low inlet velocities, the agreement improves gradually when the inlet velocity exceeds $20 \mathrm{~m} / \mathrm{s}$. At inlet velocity higher than $30 \mathrm{~m} / \mathrm{s}$, the experimental measurements are slightly overpredicted. Nevertheless, generally, the 


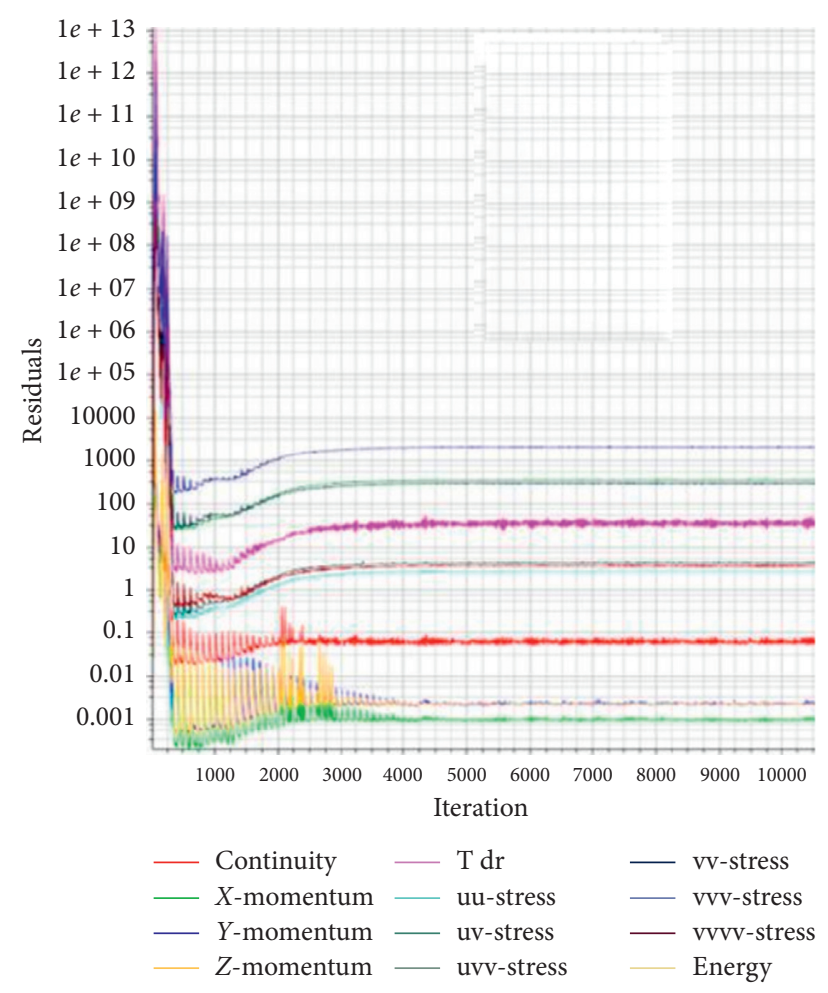

FIGURE 2: Residual function plot.

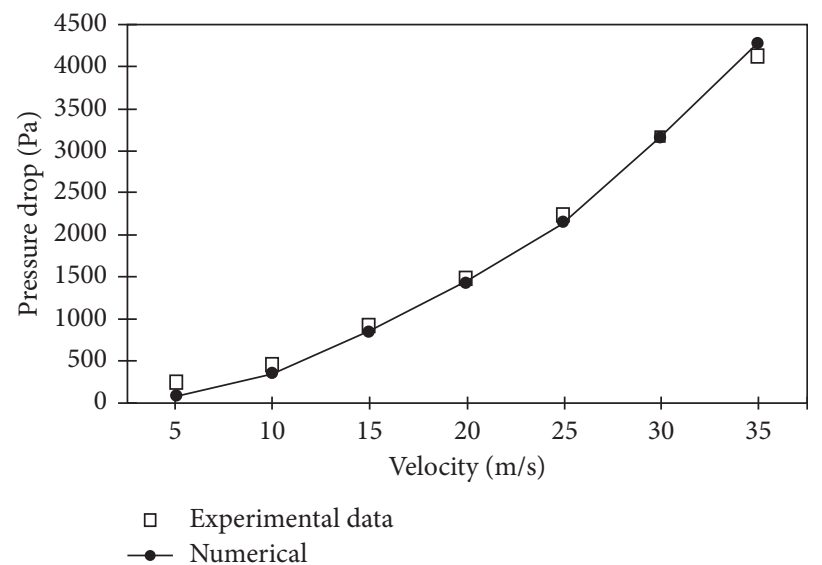

Figure 3: The computed pressure drops compared to the experimental measurements at different inlet velocities.

computed pressure drops are in good agreement with experimental measurements [17] for all inlet velocities.

From the design and efficiency point of views, the tangential velocity is critical; it aids to determine the centrifugal forces inside the separator. Accordingly, the tangential velocity is computed and compared to the experimental measurements at different axial locations inside the separator. As observed in Figure 4, the tangential velocity has an $\mathrm{M}$-shaped profile at axial locations S1, S2, and S3 while V-shaped at axial location S4. A schematic of these sections can be found in Figure 1. The tangential velocity profiles are symmetric with the axis of the separator. It is also noted that the flow creates two symmetric vortex regions known as the internal forced vortex and the external free vortex, "Rankine vortex" [23]. Typically, the tangential velocity increases with an increasing radius for the inner vortex, whereas the outer vortex decreases with an increasing radius. The computed maximum tangential velocity is approximately 1.6 the magnitude of the inlet velocity in all axial locations, which is consistent with measurements obtained by Wang et al. [17].

The contours of the tangential velocity are shown in Figure 5. The flow characteristics inside the separator are identical for inlet velocity less than $25 \mathrm{~m} / \mathrm{s}$. However, when 

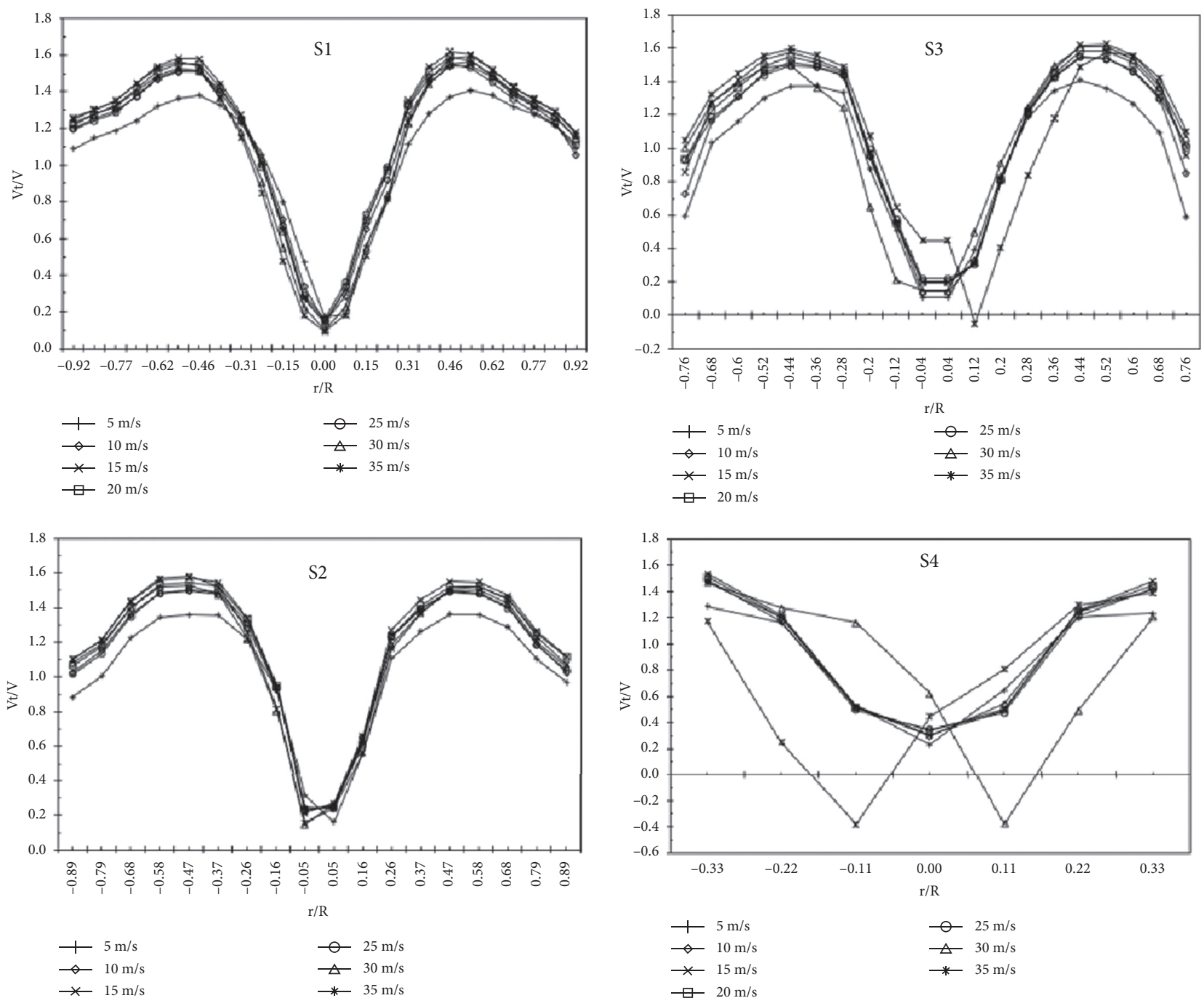

FIgURE 4: Tangential velocity profiles at different axial locations and inlet velocities.

the vortex shapes change at higher inlet velocities, the flow characteristics and flow become asymmetric due to the reverse pressure gradient in the axial position.

The axial force is vital to the separation efficiency; it affects the downstream discharge and residence time. To be noted is that the higher residence time leads to more efficient separation. The axial force pushes the particles against the wall of the separator, which increases the centripetal accelerations. With the aid of gravitational force, high forces are exerted on the particles, driving them to spin around along the walls of the separator. Figure 6 shows the axial velocity profile at different cross sections inside the separator. The inlet velocity varies from $5 \mathrm{~m} / \mathrm{s}$ to $35 \mathrm{~m} / \mathrm{s}$. At axial locations 1,2 , and 3 , the mean velocity profiles are symmetric for all inlet velocities. However, at axial location 4, there is a pattern of irregularities. Also, it was observed that the shape of the mean axial velocity varies from $\mathrm{W}$-shape at axial location 1 to $\mathrm{U}$-shape and $\mathrm{V}$-shape at axial locations 2 and 3 , respectively.

Furthermore, it is noted that there are some variations in the rotational characteristic of the flow when the velocity exceeds $30 \mathrm{~m} / \mathrm{s}$, as shown in the axial velocity contours in Figure 7. The inner vortex of the separator becomes unstable and is no longer axis symmetric near the exit of the conical part, which is due to the strong coupling between the axial and tangential velocity components and the swirls. The results also show that the axial velocity is negative in the outer vortex (from green to blue) and positive in the inner vortex (from green to red). The interface between these two vortices is located at the point where the axial velocity is zero. The distance between the vertical centre of the separator and this interface is expected to decrease along with the radius in the conical part of the separator. This is true for velocity $5-25 \mathrm{~m} / \mathrm{s}$, but not when the velocity exceeds $30 \mathrm{~m} / \mathrm{s}$.

The reason for this deviation is that when the axial velocity increases, the bottom exit is incapable of handling the increased flow, which results in an overflow. The overflow leads to an accumulation of upward flow, thus changing the radial location of the zero axial velocity interface, which is observed by the "fluctuations" in the bottom of the separator. 


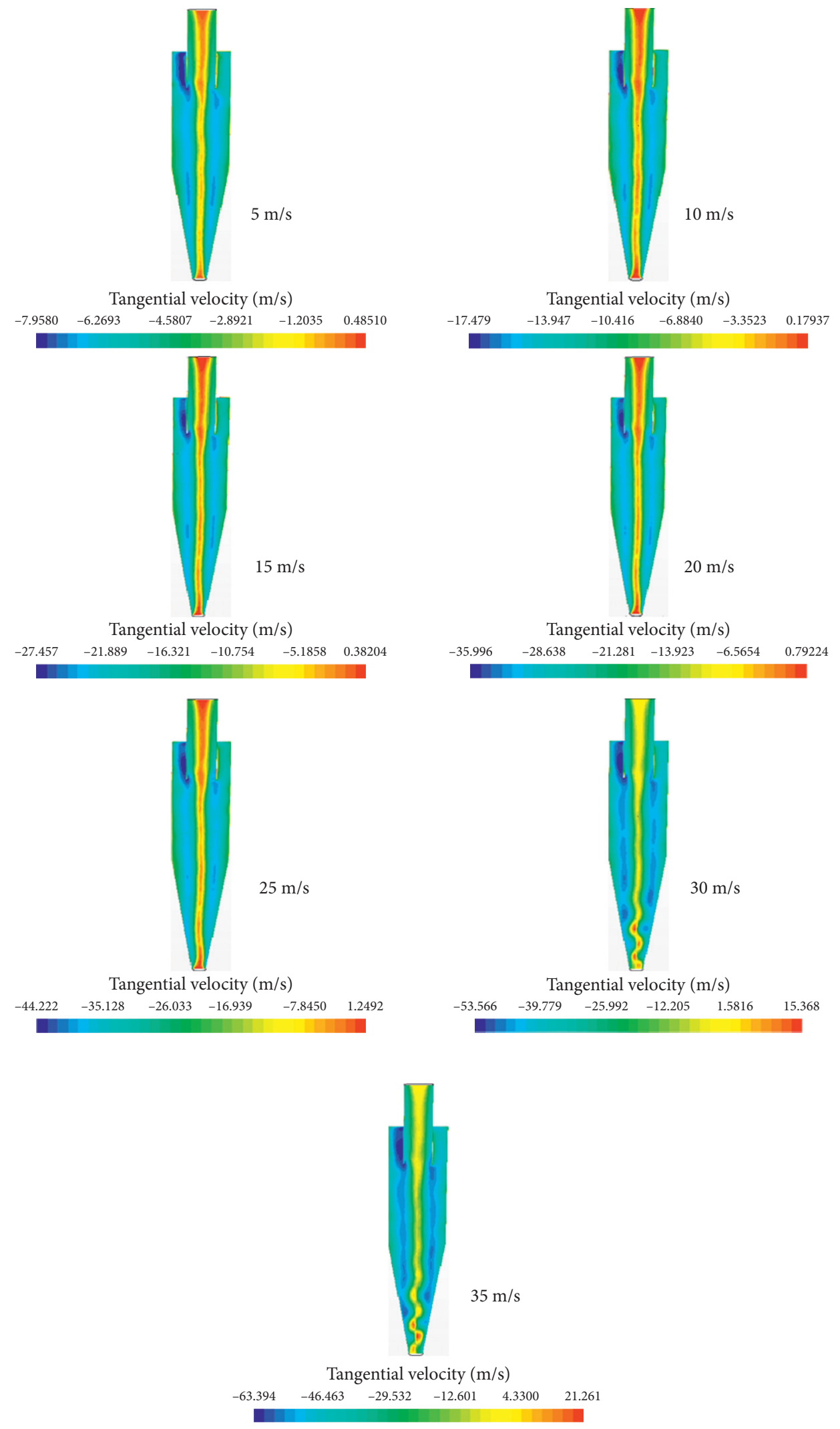

Figure 5: Tangential velocity contours at different inlet velocities. 

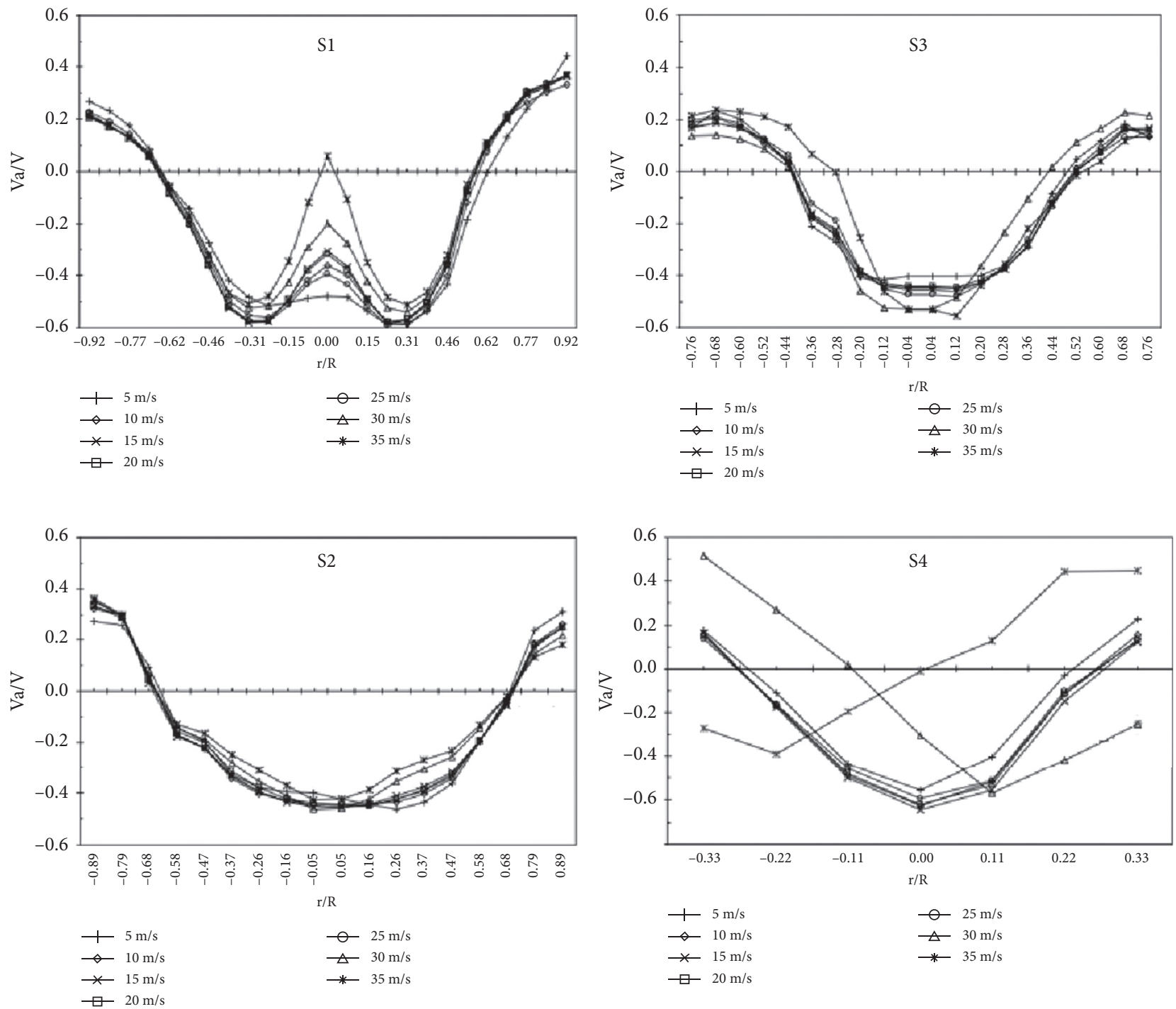

Figure 6: Axial velocity profiles for different cross sections at different inlet velocities.

One of the most significant parameters of the cyclone separators is efficiency, which is greatly influenced by both the size of the particles and the inlet velocity. Figure 8 represents the separation efficiency as a function of the diameter of the particle. Centripetal force influences larger particles by carrying them out toward the walls of the separator, which then fall downwards by gravity. Thus, as one would expect, the separation efficiency increases with increasing particle diameter, and hence, for particles with a size larger than $3 \mu \mathrm{m}$, the separation efficiency is approaching $100 \%$. The lighter particles will be captured by the inner vortex and then follow the flow to the top exit.

Figure 9 shows the separation efficiency as a function of the inlet velocities. The computed results are in acceptable agreement with the experimental measurements with an average error of less than $6 \%$. Also, the results show that separation efficiency is high for high inlet velocities because increasing inlet velocity leads to a higher centripetal acceleration acting on the particles. 


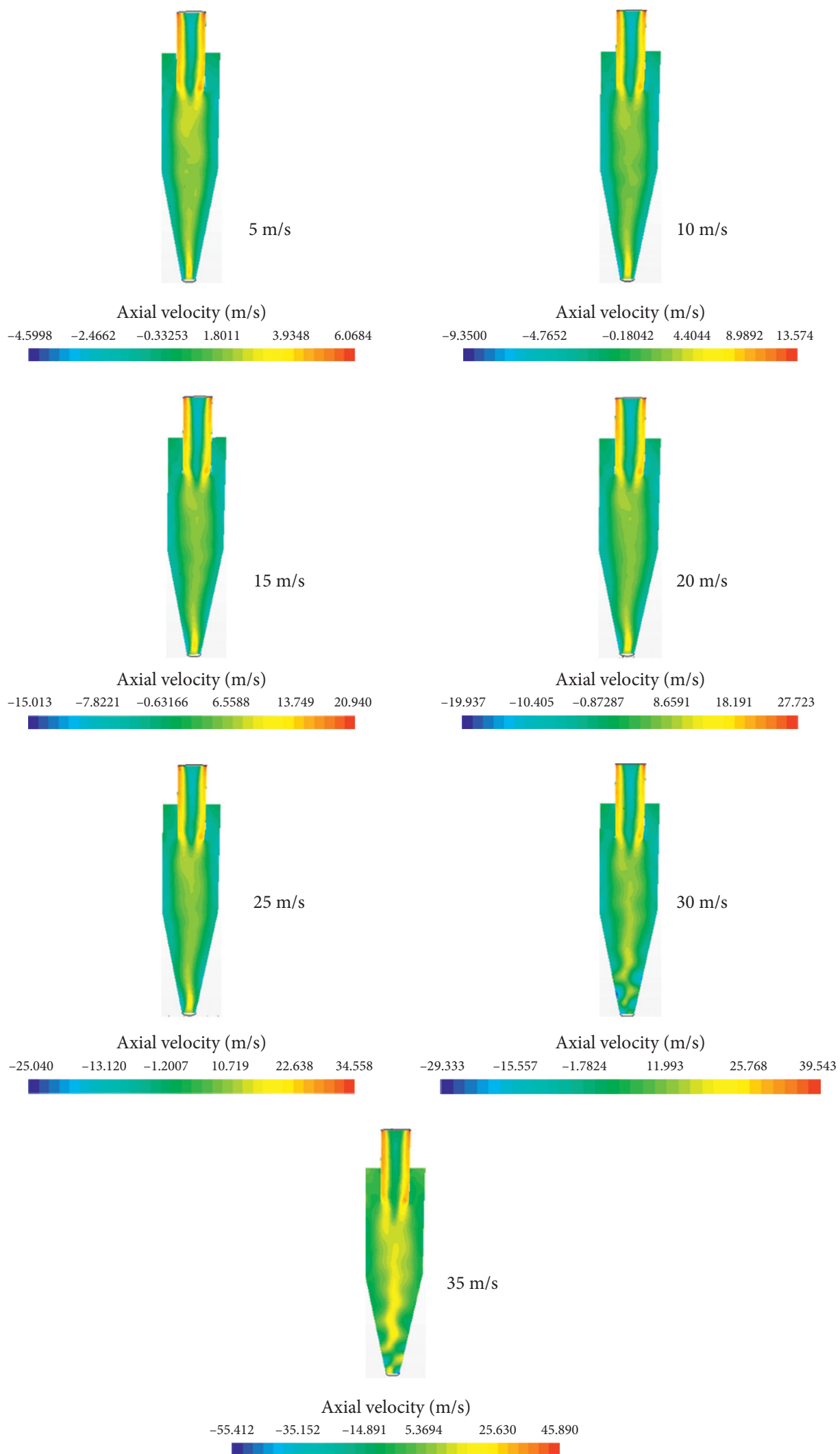

Figure 7: Axial velocity contours with different inlet velocities. 


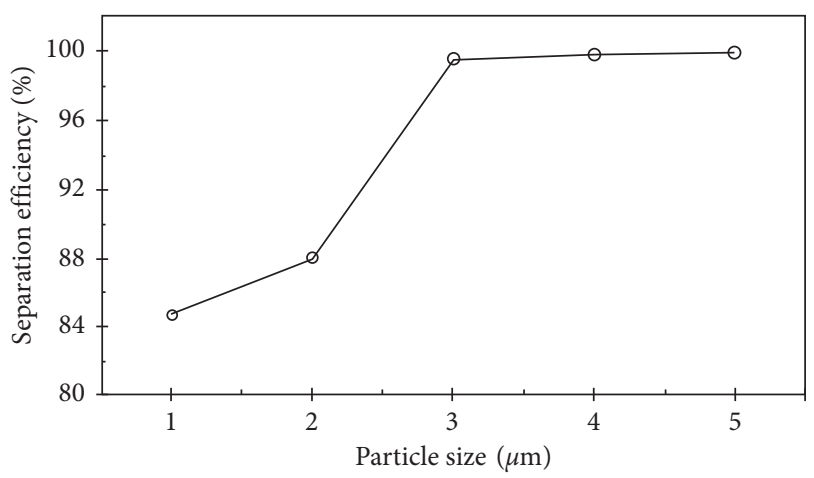

Figure 8: Particle separation efficiency as a function of particle diameter.

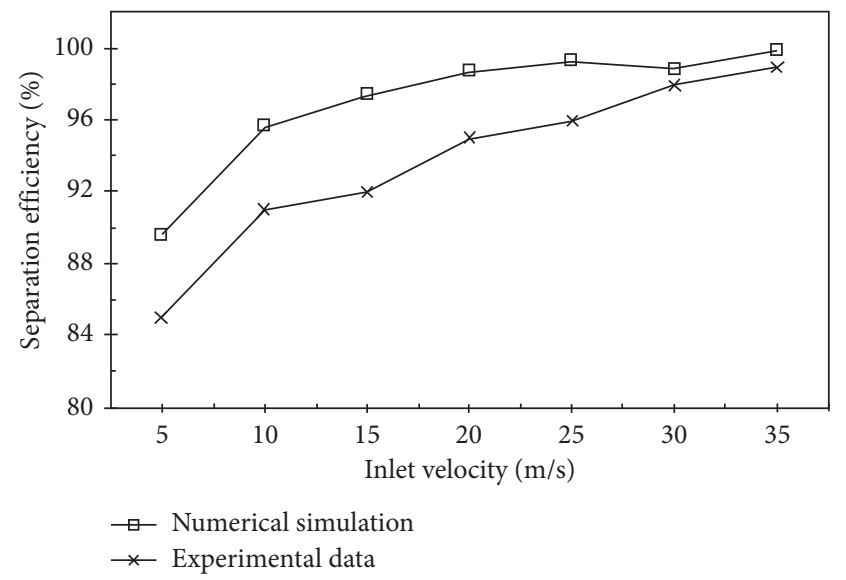

Figure 9: Particle separation efficiency as a function of inlet velocity compared with calculated results and previous simulations.

\section{Conclusion and Remarks}

This study represents a detailed two-phase numerical simulation of a cyclone separator using a multiphase Eulerian-Lagrangian gas flow method. The computational results obtained are compared to the experimental results of Wang et al. [17]. The experimental separator is used to separate air contaminated with cement particles. The contaminated air is blown into a vertical separator at a velocity of $20 \mathrm{~m} / \mathrm{s}$. The particle distribution of the cement material was expressed by the Rosin-Rammler equation [22]. The steady Favre-averaged transport equations along with their selected closures are solved on the physical grid using commercial STAR$\mathrm{CCM}+$ software. The nonlinear differential steady governing equations for the flow fields are discretised using a mixed finite element method. The Reynolds stress turbulence model is used because of the intense mixing inside the separator. The sensitivity of the computed results to the cell size and type is tested, and the presented results showed a negligible sensitivity to the number and type of cells.

The computed pressure drop was in good agreement with experimental measurements at different inlet velocities. It has been noted that the computed pressure drops increase with an increase in the inlet velocities, which reduces the separation efficiency and makes the separation operation costly. The model was also able to mimic the two distinctive symmetric vortexes inside the separator. The tangential velocity was also computed and compared to the experimental measurements at different axial locations inside the separator. It was observed that the tangential velocity has an M-shaped profile at axial locations S1, S2, and S3 while V-shaped at axial location S4.

Even though there were some minor discrepancies between the measured and computed solution, the Eulerian-Lagrangian gas flow method has successfully mimicked the experimental measurement at different axial locations inside the separator, and the general agreement was reasonably acceptable.

\section{Data Availability}

Readers can access the experimental data used in this study from [17].

\section{Conflicts of Interest}

The authors declare no conflicts of interest.

\section{References}

[1] L. Mølhave, S. K. Kjærgaard, and J. Attermann, "Sensory and other neurogenic effects of exposures to airborne office dust," Atmospheric Environment, vol. 34, pp. 4755-4766, 2000.

[2] T. Schneider, J. Sundell, W. Bischof et al., “"EUROPART”. airborne particles in the indoor environment. a European interdisciplinary review of scientific evidence on associations between exposure to particles in buildings and health effects," Indoor Air, vol. 13, no. 1, pp. 38-48, 2003.

[3] D. Bradley, "Categories of cyclone," in International Series of Monographs in Chemical Engineering, pp. 158-199, The Hydrocyclone, Pergamon, Oxford, UK, 1965.

[4] L. Svarovsky, Solid-Liquid Separation: 6 - Hydrocyclones, Butterworth-Heinemann, Oxford, UK, 4th edition, 2001.

[5] J. M. Campbell, Gas Conditioning and Processing: 2 - the Equipment Modules, Campbell Petroleum Series, Okla, $\mathrm{OH}$, USA, 7th edition, 1992.

[6] Y. Eflita, T. Mohammad, Y. Bachtiar, C. Kwang-Hwan, and P. Vita, "Effect of vortex limiter position and metal rod insertion on the flow field, heat rate, and performance of cyclone separator," Powder Technology, vol. 377, pp. 464-475, 2021.

[7] Y. Eflita, T. Mohammad, R. P. Arbian, E. D. Ade, and C. Kwang-Hwan, "Numerical analysis on the effect of the vortex finder diameter and the length of vortex limiter on the flow field and particle collection in a new cyclone separator," Cogent Engineering, vol. 5, no. 1, 2018.

[8] A. Mohsen and A. Mehdi, "An analytical study of the effect of inlet velocity on the cyclone performance using mathematical models," Powder Technology, vol. 217, pp. 121-127, 2012.

[9] F. Ficici, V. Arı, and M. Kapsiz, "The effects of vortex finder on the pressure drop in cyclone separators," International Journal of the Physical Sciences, vol. 5, pp. 804-813, 2010.

[10] E. Khairy and L. Chris, "Numerical modeling of the flow field and performance in cyclones of different cone-tip diameters," Computers \& Fluids, vol. 51, no. 1, pp. 48-59, 2011.

[11] B. Zhao, Y. Su, and J. Zhang, "Simulation of gas flow pattern and separation efficiency in cyclone with conventional single and spiral double inlet configuration," Chemical Engineering Research and Design, vol. 84, no. 12, pp. 1158-1165, 2006. 
[12] J. Houben, E. Brunnmair, C. Weiss, and S. Pirker, "CFD simulations of pressure drop and velocity field in a cyclone separator with central vortex stabilization rod," Journal of Applied Fluid Mechanics, vol. 9, no. 1, pp. 487-499, 2016.

[13] E. Brunnmair, F. Dunst, and H. Flachberger, "Neuartiger gaszyklon mit differenzierter grob- und feinabtrennzone," BHM Berg- und Hüttenmännische Monatshefte, vol. 154, no. 12, pp. 610-613, 2009.

[14] I. Karagoz, A. Avci, A. Surmen, and O. Sendogan, "Design and performance evaluation of a new cyclone separator," Journal of Aerosol Science, vol. 59, pp. 57-64, 2013.

[15] K. W. Chu, J. Chen, B. Wang et al., "Understand solids loading effects in a dense medium cyclone: effect of particle size by a CFD-DEM method," Powder Technology, vol. 320, pp. 594609, 2017.

[16] H. Yoshida, Y. Hayase, K. Fukui, and T. Yamamoto, "Effect of conical length on separation performance of sub-micron particles by electrical hydro-cyclone," Powder Technology, vol. 219, pp. 29-36, 2012.

[17] B. Wang, D. L. Xu, K. W. Chu, and A. B. Yu, "Numerical study of gas-solid flow in a cyclone separator," Applied Mathematical Modelling, vol. 30, no. 11, pp. 1326-1342, 2006.

[18] B. Guo, Petroleum Production Engineering: A ComputerAssisted Approach, Elsevier, Amsterdam, The Netherlands, 2007.

[19] B. Andersson, R. Andersson, L. Håkansson, M. Mortensen, R. Sudiyo, and B. V. Wachem, Computational Fluid Dynamics for Engineers, Cambridge University Press, Cambridge, UK, 2011.

[20] A. Faghri and Y. Zhang, Transport Phenomena in Multiphase Systems: 4 - Generalised Governing Equations for Multi-phase Systems: Averaging Formulations, Academic Press, Boston, MA, USA, 2006.

[21] S. Subramaniam, "Lagrangian-Eulerian methods for multiphase flows," Progress in Energy and Combustion Science, vol. 39, 2012.

[22] A. H. Nissan and V. P. Bresan, "Swirling flow in cylinders," AIChE Journal, vol. 7, no. 4, pp. 543-547, 1961.

[23] S. V. Alekseenko, P. A. Kuibin, V. L. Okulov, and S. I. Shtork, "Swirling flow in cylinders," AIChE Journal, vol. 7, no. 4, pp. 543-547, 1961.

[24] S. V. Alekseenko, P. A. Kuibin, V. L. Okulov, and S. I. Shtork, "Helical vortices in swirl flow," Journal of Fluid Mechanics, vol. 382, pp. 195-243, 1999. 